\title{
Elevated Airway Eosinophils is Associated with Lower Airway Haemophilus
}

\author{
Vyvian C. Borse ${ }^{1}$, Sydney E. Ross $\mathrm{MS}^{2}$, Alexis A. McEntire BS ${ }^{2}$, James Slaven \\ $\mathrm{MS}^{3}$, and Kirsten M. Kloepfer MD MS ${ }^{2}$ \\ ${ }^{1}$ Indiana University School of Medicine, ${ }^{2}$ Section of Pulmonary, Allergy, and \\ Sleep Medicine, Department of Pediatrics, Indiana University School of Medicine, \\ and ${ }^{3}$ Department of Biostatistics, Indiana University School of Medicine
}

Background and Hypothesis: Early wheezing and persistent cough in young children can be a first indication of asthma. Studies show that infants later diagnosed with asthma commonly exhibit airway microbiome dysbiosis particularly with pathogenic bacteria. Two such bacteria, Moraxella catarrhalis and Haemophilus influenzae, can stimulate pro-inflammatory cytokines such as IL-5. Because IL-5 can signal eosinophil production, we hypothesize children with asthma and positive bronchoalveolar lavage fluid (BALF) culture will have elevated BALF eosinophils.

Project Methods: Children undergoing clinically indicated bronchoscopy in the Riley Hospital outpatient center were recruited. Chart review was conducted to examine associations between BALF culture, BALF differential cytology, diagnoses, antibiotic history, and demographic data from biobank participants. Exclusion criteria included: diagnosis of cystic fibrosis or pulmonary ciliary dyskinesia; BALF culture not performed; and/or hypocellular sample. Statistical analyses included Student's t-test, Wilcoxon rank-sum tests, and Chi-Square tests (verified with Fisher's Exact tests).

Results: 94 samples were analyzed ( $37 \%$ female, age: 1 mos $-17 \mathrm{yrs}$ ). BALF was positive for the following bacteria: $M$. catarrhalis (26\%), $H$. influenzae (28\%), S. pneumoniae $(27 \%)$, and S. aureus $(17 \%)$. Haemophilus in BALF was associated with elevated eosinophils $(p=.03)$ and neutrophils $(p=.04)$ and decreased macrophages $(p=.001)$. Asthma diagnosis and prior antibiotic use were not significantly associated with positive BALF culture.

Conclusion and Potential Impact: Haemophilus was associated with elevated eosinophils in the airway. The impact of this observation remains unclear. Further research is necessary to determine pathways that lead to these observed changes within the airway. 